\title{
Effect of rehabilitation on the somatosensory evoked potentials and gait performance of hemiparetic stroke patients
}

\author{
Hyun S. Yoon ${ }^{\mathrm{a}, \mathrm{b}}$, Young J. Cha ${ }^{\mathrm{b}}$, Min K. Sohn ${ }^{\mathrm{a}}$ and Joshua (Sung) H. You ${ }^{\mathrm{b}, *}$ \\ ${ }^{a}$ Chungnam National University Hospital, Daejeon, Korea \\ ${ }^{\mathrm{b}}$ Sports $\cdot$ Movement Institue and Technology, Department of Physical Therapy Program, Yonsei \\ University, Wonju, Korea
}

\begin{abstract}
.
BACKGROUND: Gait performance of stroke patients is affected by impaired sensory ability. The purpose of the present study was to determine the relationship between somatosensory-evoked potential (SSEP) parameters and gait performance in hemiparetic stroke patients.

METHODS: A convenience sample of 17 hemiparetic stroke patients (mean age $60.11 \pm 8.83$ years; 10 women; right hemiplegia: 10, left hemiplegia: 7) were recruited for the present study. The Electro Synergy system (Viasys Healthcare; San Diego, CA, USA) was used for SSEP evaluation. The 17 patients were assigned to two groups according to their SSEP results as follows: 8 patients to the normal response group and 9 patients to the abnormal group. All the participants underwent the same rehabilitation exercise programs during 4 weeks, followed by clinical evaluation. A mixed-design analysis of a variance model was used to test for differences in timed up-and-go (TUG) test and 10-meter walking test (10MWT) scores between the two independent groups while the participants were subjected to repeated measures (pretest and posttest).

RESULTS: Analysis of variance revealed the main time effect $(p<0.05)$ and group by time interaction effect $(p<0.05)$. The post hoc test result confirmed that the normal sensory group showed greater improvement in TUG test and 10MWT scores than the abnormal sensory group $(p<0.05)$. The TUG test and 10MWT scores in the posttest were greater in the normal sensory group than in the abnormal sensory group.

CONCLUSIONS: The present study demonstrated the importance of the clinical contribution of the baseline sensory function of individuals with hemiparetic stroke to their gait performance and recovery after stroke rehabilitation. As anticipated, the individuals who had intact or spared sensory function showed greater improvements in gait speed and performance measures than those who had impaired sensory function.
\end{abstract}

Keywords: Stroke, somatosensory-evoked potential, balance, gait

\section{Introduction}

The sensorimotor function of the central nerve system (CNS) is important for balance reaction and ambulation and often impaired in hemiparetic stroke after the development of a CNS lesion [1]. Among

\footnotetext{
${ }^{*}$ Corresponding author: Joshua (Sung) H. You, Department of Physical Therapy, Yonsei University Director of Sports . Movement Institue and Technology (S·MIT), 1 Yonseidae Kil, Wonju City, Kangwon-do 220-710, Korea. Tel.: +82 33760 2476; Fax: +82 33760 -2496; E-mail: neurorehab@yonsei.ac.kr.
}

0928-7329/18/\$35.00 (c) 2018 - IOS Press and the authors. All rights reserved

This article is published online with Open Access and distributed under the terms of the Creative Commons Attribution NonCommercial License (CC BY-NC 4.0). 
the various sensory inputs, including visual, proprioceptive, and vestibular information, the somatosensory information, which provide joint position and kinesthetic movement sense, is the most essential for symmetrical balance and locomotor movement control [2]. Conventionally, clinical sensory measurement was used to detect the degree of sensory impairment in neurological patients such as those who had a stroke, spinal cord injury, and multiple sclerosis. However, due to measurement sensitivity and reliability issues, the somatosensory-evoked potential (SSEP) has been used recently to determine the sensory integrity associated with cutaneous and proprioceptive sensory function. SSEP measures the electrophysiological response of the nervous system to a standardized electrical sensory stimulation, which is measured along the sensory pathway (i.e., posterior tibial nerve) [3]. Neurophysiologically, the posterior tibial nerve stimulation elicits the large-diameter, fast-conducting Ia muscle afferent and group II cutaneous nerve fibers, with which orthodromic sensory stimulation generates the SSEP [3]. The neural activity from stimulation transmits sensory signals associated with proprioception and vibration that ascends the ipsilateral dorsal column and medial lemniscus pathways to the frontoparietal sensory motor cortex [4]. Hence, the post-stimulus latency of an SSEP peak characterizes neurological functional impairments as measured by peak waveform amplitude, conduction velocity (CV), and central conduction time (CCT), which result from the stimulation of a nerve [5]. Tibial nerve SSEP is generated by the primary somatosensory cortex, which subserves detecting the ischemic stroke events associated with anterior cerebral artery aneurysm. Therefore, it is clinically useful to monitor the ischemic insult associated with cerebral aneurysm surgery, or prognostic outcomes in balance and gait performance after stroke [6]. However, it is unclear how the SSEP can be used to predict or quantify the degree of the balance and gait function of stroke patients, although previous studies showed potential correlations between SSEP and gait recovery [7,8]. The purpose of the present study was to determine the relationship between SSEP parameters and gait performance (timed up and go [TUG] test and 10-meter walking test [10MWT]) parameters in stroke patients. Our hypothesis was that an association exists between SSEP and gait performance parameters.

\section{Materials and methods}

\subsection{Materials and procedure}

A convenience sample of 17 adults with hemiparetic stroke (mean age $60.11 \pm 8.83$ years; 10 women; right hemiplegia: 10; left hemiplegia: 7) were recruited for the present study. The diagnosis of stroke was based on clinical history and examination results, and confirmed by using computed tomography or magnetic resonance imaging(supratentorial lesion: 12, infratentorial lesion: 5; infarction: 11, hemorrhage: 6). All the participants underwent a consistent rehabilitation exercise program for 45 minutes a day, 3-5 times a week over a 4-week period. The subjects who had a subacute hemiplegic stroke (post-stroke onset duration $<3$ months; mean months $1.82 \pm 0.72$ ) and could follow the instruction (Mini-Mental State Examination-Korea [MMSE-K] mean score $27.23 \pm 1.56$ ) were included. However, stroke patients with severe visual hemianopia, cognitive impairments, sensory neuropathy, or previous surgical history were excluded. The Electro Synergy system (Viasys Healthcare; San Diego, CA, USA) was used to examine the baseline SSEP parameters prior to rehabilitation. A bar electrode was used to stimulate the paretic side posterior tibial nerve. The cathode was positioned in the middle site between the medial border of the Achilles tendon and medial malleolus posterior border, and stimulated the posterior tibial nerve at $30 \mathrm{~mA}$. The anode was located $3 \mathrm{~cm}$ distally from the cathode. The signals were recorded with fine-needle electrodes by inserting a reference electrode in the Fz and the active electrode 


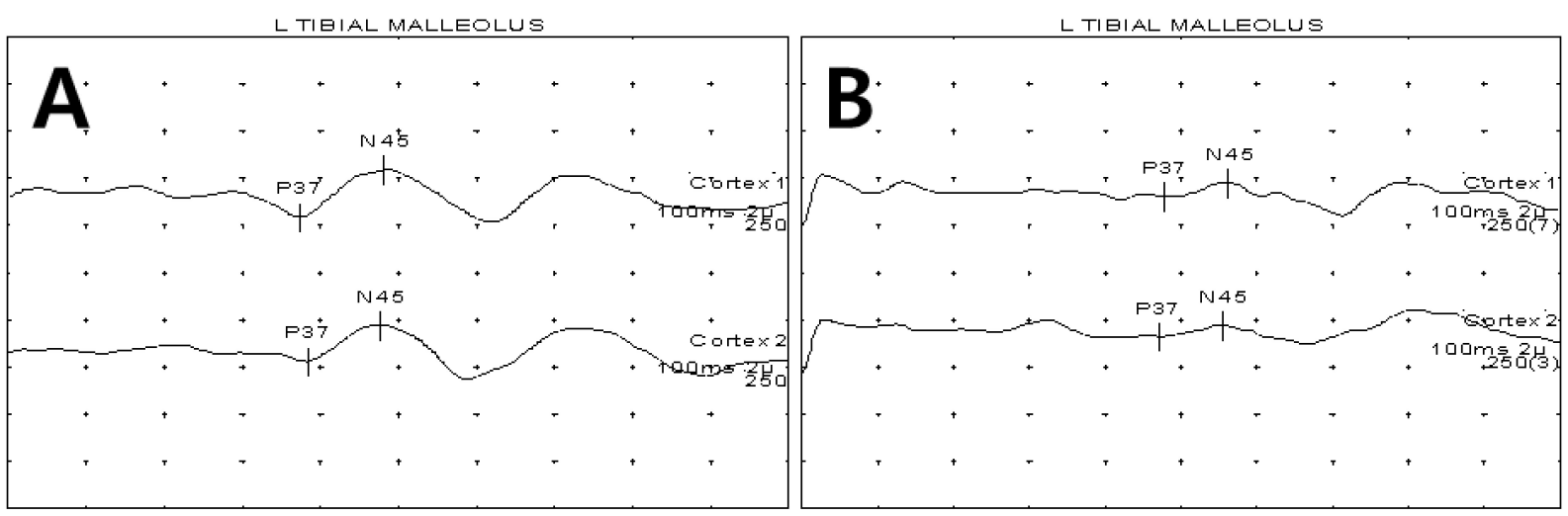

Fig. 1. Examples of SSEP graphs for patients with normal (A) and abnormal SSEPs (B). SSEP data were classified into (A) a normal sensory group (normal latency; P37 $<41.7 \mathrm{~ms}$ ) and (B) an abnormal sensory group (delayed latency; P37 $\geqslant 41.7 \mathrm{~ms}$ ). SSEP: somatosensory-evoked potential.

in $\mathrm{Cz}$ as per the international 10-20 system [8]. Two repetitive stimuli were produced with a frequency of 2.3 per second for 250 pulses and were averaged. Band filter width was set to 20-2000 Hz. SSEP was considered normal if P37 was $<41.7 \mathrm{~ms}$ and abnormal if it was $\geqslant 41.7 \mathrm{~ms}$ [9]. Based on the baseline SSEP data, 8 patients were classified into the normal sensory response group (NSG) and 9 patients into the abnormal sensory group (ASG; Fig. 1). The clinical test included the standardized TUG test and 10MWT. The TUG test and 10MWT are reliable and valid measurement tools to assess dynamic standing balance and gait speed in older adults $[10,11]$. The tests require performing tasks that consist of standing up from a chair, walking $3 \mathrm{~m}$, returning to the chair, and sitting down and checking the time to complete the test [12]. A previous study suggested that all healthy community-dwelling subjects aged $65-84$ years performed the test in $<20$ seconds [13]. In the 10MWT, each patient was asked to walk as quickly as possible [14].

\subsection{Statistical analysis}

Descriptive statistical data analyses included means and standard deviation. A mixed-design analysis of a variance model was used to test for differences in TUG test and 10MWT scores between the two independent groups (NSG and ASG) while the participants were subjected to repeated measures (pretest and posttest). The Tukey post hoc test was performed when the inter-action effects were observed. Pearson's correlation was analyzed a relationship between the SSEP and TUG/10MWT.

\section{Results}

\subsection{TUG (Dynamic balance and gait speed)}

Analysis of variance (ANOVA) revealed the main time effect $(p<0.05)$ and group by time interaction effect $(p<0.05)$. The post hoc test confirmed that the NSG showed greater improvement in TUG test score than the ASG $(p<0.05)$. The TUG test score in the posttest was greater in the NSG than in the ASG (Table 1).

\section{2. $10 M W T$ (Gait speed)}

The ANOVA revealed the main time effect $(p<0.05)$ and group by time interaction effect $(p<0.05)$. 
Table 1

Gait performance data between the NSG and ASG of stroke patients in the study

\begin{tabular}{|c|c|c|c|c|}
\hline Test & & NSG $(n=8)$ & ASG $(n=9)$ & Post hoc \\
\hline \multirow[t]{4}{*}{ TUG } & Pretest & $46 \pm 26.29$ & $42.55 \pm 17.23$ & \\
\hline & Posttest & $15.62 \pm 5.99$ & $32.66 \pm 14.72$ & $0.01^{\dagger}$ \\
\hline & Time, $p$-value & & & \\
\hline & Time $\times$ group, $p$ value & & & \\
\hline \multirow[t]{4}{*}{ 10MWT } & Pretest & $40.25 \pm 20.85$ & $39.11 \pm 15.46$ & \\
\hline & Posttest & $14.62 \pm 6.13$ & $31 \pm 15.75$ & $0.01^{\dagger}$ \\
\hline & Time, $p$ value & & & \\
\hline & Time $\times$ group, $p$ value & & & \\
\hline
\end{tabular}

TUG: Timed up-and-go test; 10MWT: 10-meter walking test; the values are presented as mean \pm SD; NSG: normal sensory group; ASG: abnormal sensory group. ${ }^{*} p<0.05$, derived from the main and interaction effects. ${ }^{\dagger} p<0.01$, derived from the post hoc test (Tukey's honest significant difference).

The post hoc test confirmed that the NSG showed greater improvement in 10MWT score than the ASG $(p<0.05)$. The 10MWT score in the posttest was greater in the NSG than in the ASG (Table 1).

\subsection{Relationship between the SSEP and TUG/10MWT}

Pearson's correlation analysis showed a strong relationship between the SSEP and TUG $(r=0.66$, $p<0.01)$ as well as between the SSEP and a 10MWT score $(r=0.61, p<0.01)$.

\section{Discussion}

The present study demonstrated the importance of the clinical contribution of the baseline sensory function of individuals who had a hemiparetic stroke to their gait performance and recovery after stroke rehabilitation. As anticipated, the individuals who had an intact or preserved sensory function showed greater improvements in gait speed and performance measures than those who had impaired sensory function. Moreover, the correlation analysis showed a strong relationship between the SSEP and TUG and 10MWT, suggesting a predictive relationship between the sensory integrity and gait outcome variables. These findings are consistent with those of a previous study [15] that examined the relationship between the integrity of the tibial nerve SSEP and the extent of recovery of motor function in terms of Fugl-Meyer assessment (FMA) score. Kim and colleagues [15] reported that individuals with higher or normal sensory functions showed greater recovery of motor function assessed by FMA score than those with impaired sensory function after stroke rehabilitation.

Functional locomotor recovery in hemiparetic stroke has been attributed to multiple factors, including sensorimotor dysfunction, muscle imbalance, asymmetric or unilateral muscle weakness, spasticity, and movement coordination [16]. Among these factors, sensorimotor dysfunction influences muscle imbalance and movement coordination, which consist of postural control and locomotion [17]. Altered somatosensory input (joint position sense and proprioception) transmitted from the involved limb to the primary motor cortex via ascending pathways can generate inappropriate motor output to the spinal cord via descending pathways [18], which convey locomotor output signals through alpha and gamma motor neurons, which innervate the lower extremity muscles for walking [19]. Somatosensory proprioception has been identified as an important afferent input for the efficient locomotor control and recovery, as it is involved in the feedback motor control mechanism in the gait and posture in stroke rehabilitation [20]. 
In fact, the increasing gait speed between SSEP and walking function in the present study further support this notion. Lastly, one limitation of the present study is that the results should be interpreted with caution when generalizing our findings to chronic hemiparetic populations. Future research should be conducted with a larger sample size to increase the experimental robustness and generalizability of our findings.

\section{Conclusion}

The present study demonstrated the importance of the clinical contribution of the baseline sensory function of individuals who had a hemiparetic stroke to their gait performance and recovery after stroke rehabilitation. As anticipated, the individuals who had an intact or preserved sensory function showed greater improvements in gait speed and performance measures than those with impaired sensory function. These results suggest that the degree of posterior tibial nerve damage can contribute to gait performance and speed. Clinically, this study provides important insight for increasing gait performance.

\section{Acknowledgments}

This study was supported by the "Brain Korea 21 PLUS Project" (grant No. 2016-51-0009), sponsored by the Korean Research Foundation for the Department of Physical Therapy, Graduate School, Yonsei University.

\section{Conflict of interest}

None to report.

\section{References}

[1] Geurts AC, de Haart M, van Nes IJ, Duysens J. A review of standing balance recovery from stroke. Gait \& Posture. 2005; 22(3): 267-81.

[2] Belda-Lois JM, Mena-del Horno S, Bermejo-Bosch I, Moreno JC, Pons JL, Farina D, Caria A. Rehabilitation of gait after stroke: a review towards a top-down approach. Journal of Neuroengineering and Rehabilitation. 2011; 8(1): 66.

[3] Regan D. Some characteristics of average steady-state and transient responses evoked by modulated light. Electroencephalography and Clinical Neurophysiology. 1966; 20(3): 238-48.

[4] Nuwer MR. Fundamentals of evoked potentials and common clinical applications today. Electroencephalography and Clinical Neurophysiology. 1998; 106(2): 142-48.

[5] Colon E, Visser SL. Evoked potential manual: a practical guide to clinical applications. Springer Science \& Business Media. 2012.

[6] Lee SY, Kim BR, Han EY. Association between evoked potentials and balance recovery in subacute hemiparetic stroke patients. Annals of Rehabilitation Medicine. 2015; 39(3): 451-61.

[7] Al-Rawi MA, Hamdan FB, Abdul-Muttalib AK. Somatosensory evoked potentials as a predictor for functional recovery of the upper limb in patients with stroke. Journal of Stroke and Cerebrovascular Diseases. 2009; 18(4): 262-8.

[8] Tzvetanov P, Rousseff RT, Atanassova P. Prognostic value of median and tibial somatosensory evoked potentials in acute stroke. Neuroscience Letters. 2005; 380(1): 99-104.

[9] Miura T, Sonoo M, Shimizu T. Establishment of standard values for the latency, interval and amplitude parameters of tibial nerve somatosensory evoked potentials (SEPs). Clinical Neurophysiology. 2003; 114(7): 1367-78.

[10] Shumway-Cook A, Brauer S, Woollacott M. Predicting the probability for falls in community-dwelling older adults using the timed up and go test. Physical Therapy. 2000; 80(9): 896. 
[11] Steffen TM, Hacker TA, Mollinger L. Age- and gender-related test performance in community-dwelling elderly people: six-minute walk test, Berg Balance Scale, timed up and go test, and gait speeds. Physical Therapy. 2002; 82(2): 128.

[12] Podsiadlo D, Richardson S. The timed "up and go": a test of basic functional mobility for frail elderly persons. Journal of the American Geriatrics Society. 1991; 39(2): 142-8.

[13] Bohannon RW. Reference values for the timed up and go test: a descriptive meta-analysis. Journal of Geriatric Physical Therapy. 2006; 29(2): 64-8.

[14] Deathe AB, Miller WC. The L test of functional mobility: measurement properties of a modified version of the timed "up and go" test designed for people with lower-limb amputations. Physical Therapy. 2005; 85(7): 626.

[15] Kim SW, Kim SB, Lee SY, Koh SE, Lee JM, Lee JY. Motor evoked potentials and somatosensory evoked potentials of upper and lower extremities for prediction of functional recovery in stroke. Brain \& Neurorehabilitation. 2012; 5(1): 24-31.

[16] Li S, Francisco GE. New insights into the pathophysiology of post-stroke spaticity. Frontier in human Neuroscience. 2015; 9: 192.

[17] Smania N, Picelli A, Gandolfi M, Fiaschi A, Tinazzi M. Rehabilitation of sensorimotor integration deficits in balance impairment of patients with stroke hemiparesis: a before/after pilot study. Neurological Sciences. 2008; 29(5): 313-9.

[18] Canedo A. Primary motor cortex influences on the descending and ascending systems. Progress in Neurobiology. 1997; 51(3): 287-335.

[19] Ellaway PH, Taylor A, Durbaba R. Muscle spindle and fusimotor activity in locomotion. Journal of Anatomy. 2015; 227(2): 157-166.

[20] Riemann BL, Lephart SM. The sensorimotor system, Part II: the role of proprioception in motor control and functional joint stability. Journal of Athletic Training. 2002; 37: 80-4. 